\title{
Corneal Langerhans cell and dry eye examinations in ankylosing spondylitis
}

\author{
László Marsovszky', János Németh', Miklós D Resch', \\ Gergely Toldi ${ }^{2}$, Nóra Legány ${ }^{3}$, László Kovács ${ }^{3}$ and Attila Balog ${ }^{3}$
}

\begin{abstract}
APCs of the ocular surface, including corneal Langerhans cells (LCs), offer the opportunity to gain insight into the activity of innate immunity. We examined corneal LCs and dry eye parameters in ankylosing spondylitis (AS). Twenty-four AS patients with varying degrees of disease activity and 24 healthy participants were enrolled. Central and peripheral LC numbers, and Langerhans cell morphology (LCM) were assessed with in vivo laser confocal microscopy. In addition, ocular surface disease index, lid parallel conjunctival folds, tear break up time, and Schirmer test were evaluated. LC densities and central LCM were greater in AS patients than in the controls. Moreover, LCM was significantly greater in patients with higher systemic inflammation according to elevated C-reactive protein (CRP). Also, tear production was greatly suppressed in patients with more severe onset of the systemic inflammation according to the Bath Ankylosing Spondylitis Disease Activity Index and elevated CRP. Greater corneal LC density and LCM in AS may reflect an increased activation state of the innate immune system of the cornea in AS, which correlates with the systemic activity of AS even without ocular symptoms. Nonetheless, higher systemic inflammation might impair tear production, and it might partly explain the dry eye mechanism.
\end{abstract}

\section{Keywords}

Corneal Langerhans cell, in vivo confocal microscopy, dry eye, ankylosing spondylitis

Date received: 18 February 20I3; revised: 21 May 20I3; accepted: 24 June 2013

\section{Introduction}

Ankylosing spondylitis (AS) is an immune-mediated rheumatic disease characterized by chronic inflammation. The autoimmune reaction principally affects the axial and sacroiliac joints in AS. As the disease progresses, the chronic inflammation of the spine (spondylitis) causes extra bone formation and eventually leads to the fusion of the vertebrae (ankylosis). In any stage of the disease, systemic autoimmune reactions can result in significant target organ damage, causing peripheral arthritis, enthesitis and extra-articular manifestations, such as inflammation in the eye, the gastrointestinal tract and the heart. ${ }^{1}$ The most common inflammatory ophthalmic condition is uveitis, affecting $10-50 \%$ of AS patients, ${ }^{2-4}$ and Sjögren's syndrome (SS) can occur in $10 \% .{ }^{5}$ Corneal involvement is rare, and, to date, there is no substantial evidence on the activity of local corneal immune system in AS. The introduction of anti-TNF- $\alpha$ agents into the therapeutic armamentarium of AS has significantly reduced the clinical signs and symptoms of the disease, and has been used with success in uveitis refractory to the conventional treatment regime. ${ }^{6}$ It is known from previous studies that corneal Langerhans cells (LCs) are deeply involved in the corneal immunoregulatory processes. Besides being an APC of the ocular surface, they are proposed to play a role in the inflammatory processes of the ocular surface. ${ }^{7}$ Lin et al. ${ }^{8}$ have brought to light that LCs are present in greater numbers both in SS- and non-SS-associated dry eye. Our team has recently demonstrated in rheumatoid arthritis (RA) patients that LC presence was increased in the cornea and

\footnotetext{
'Department of Ophthalmology, Semmelweis University Budapest, Budapest, Hungary

${ }^{2}$ First Department of Pediatrics, Semmelweis University Budapest, Budapest, Hungary

${ }^{3}$ Department of Rheumatology, Faculty of Medicine, Albert SzentGyörgyi Health Center, University of Szeged, Szeged, Hungary
}

\section{Corresponding author:}

László Marsovszky, Department of Ophthalmology, Semmelweis University Budapest, Mária u. 39, H-I085. Budapest, Hungary.

Email: marsovsz@hotmail.com 
they were noted to be of active phenotype. ${ }^{9}$ Our objective for this present study was to further examine this concept in another inflammatory rheumatic disease, that is, AS.

\section{Materials and methods}

The study was performed in accordance with the ethical standards set out in the 1964 Declaration of Helsinki.

\section{Patients}

Twenty-four AS patients with variable severity of disease were studied with analysis of 24 eyes (see clinical characteristics in Table 1). AS patients were diagnosed according to the modified New York criteria. ${ }^{10}$ All AS patients were on a stable anti-TNF- $\alpha$ therapy, which means that the duration of the same anti-TNF- $\alpha$ medication was more than 3 months. Fifteen out of 24 AS patients $(62 \%)$ received additional nonsteroidal antiinflammatory drugs, whilst six $(25 \%)$ received sulphasalazin. Bath Ankylosing Spondylitis Disease Activity Index (BASDAI) was calculated at the time of the eye examination. BASDAI is a six-item questionnaire that indicates the activity of the illness, and is the most widely-used tool for the assessment of disease activity in AS. ${ }^{11}$ At the time of evaluation of confocal microscopic investigation, none of the AS patients suffered any overt ocular symptom requiring specific ophthalmic assistance. Patients with diabetes; previous eye surgery; uveitis within one year prior to examination; glaucoma; congenital, mechanical or toxic injury of the cornea; or illnesses causing corneal oedema, haze or scars were excluded. Secondary SS was evaluated according to the revised version of the AmericanEuropean Consensus Criteria for SS. ${ }^{12}$

Table I. Clinical characteristics of healthy individuals and AS patients.

\begin{tabular}{|c|c|c|}
\hline Characteristics & $\begin{array}{l}\text { Healthy individuals } \\
(n=24)\end{array}$ & $\begin{array}{l}\text { AS patients } \\
(n=24)\end{array}$ \\
\hline Age (years) & $52.5[34-64]$ & $39.5[34-52]$ \\
\hline Gender (male/female) & $19 / 5$ & $21 / 3$ \\
\hline AS duration (years) & - & $9[7-13]$ \\
\hline BASDAI & - & $3.2[1.5-4.4]$ \\
\hline $\begin{array}{l}\text { No. of HLA-B27-positive } \\
\text { patients }\end{array}$ & - & $12(50 \%)$ \\
\hline $\begin{array}{l}\text { No. of patients with } \\
\text { history of uveitis }\end{array}$ & - & 7 (29\%) \\
\hline CRP $(\mathrm{mg} / \mathrm{l})$ & $3.2[2.2-4.2]$ & $12.0 *[2.5-30.6]$ \\
\hline
\end{tabular}

Data are expressed as median [interquartile range]. $* P<0.05$ vs healthy individuals.
Healthy volunteers comprised 24 participants who had been free from any ocular or adnexal inflammation at least one year prior to enrolment.

\section{Examination of dry eye-related parameters}

The protocols for these procedures have been described in great detail in our previous work. ${ }^{9}$ We will summarise them here in short, and in investigational order. The Ocular Surface Disease Index (OSDI) questionnaire was used to describe subjective eye-related discomfort. This 12-item scale was specially designed for the assessment of symptoms related to dry eye disease and its effect on vision. ${ }^{13}$ Lid parallel conjunctival folds (LIPCOF) were evaluated at the temporal aspect of the lower eyelid margin according to the protocol specified by Pult et al. ${ }^{14}$ A Schirmer test (ST) was carried out without anesthesia with a standardized ST strip (Ref:4701001; Haag-Streit, Harlow, UK). Wetting of the test strip represented tear production and was considered as ST result. Patients with an ST result of less than $5 \mathrm{~mm} / 5 \mathrm{~min}$ were not automatically excluded from the study if the results of other functional tests and minor salivary biopsy were normal and no anti-Ro(SSA) or anti-La(SSB) Abs were detected. Tear break up time (TBUT) described the interval between the last complete blink and the first appearance of corneal black spot in seconds, and the mean value of three consecutive measurements was interpreted as TBUT.

\section{In vivo confocal cornea microscopy investigations}

Image acquisition. In vivo confocal scanning laser cornea microscopy was carried out with Heidelberg Retina Tomograph with Rostock Cornea Module (HRTII/ RCM) (Heidelberg Engineering, Heidelberg, Germany) on all participants after application of topical anesthetic drops (Oxybuprocaine-Humacain 0.4\%; Human Pharmaceuticals, Gödöllö , Hungary). A polymethyl-methacrylate cap (TomoCap; Heidelberg Engineering) was used to cover the immersion lens of the microscope to keep the distance from the corneal surface stable. Carbomer gel (Vidisic; Dr Mann Pharma, Berlin, Germany) was used as the coupling medium to ensure airless contact between the plastic cap and the objectives of the microscope. HRTII/ RCM uses a diode laser beam with a wavelength of $670 \mathrm{~nm}$ to scan the focal plane of the specimen examined. The inbuilt digital micrometre gauge ensured visualization of the depth of examination field in the cornea. The microscope has been designed to capture two-dimensional images with a resolution of $384 \times 384$ pixels covering an area of $400 \times 400 \mu \mathrm{m}$. Forty consecutive images of the right cornea in each group were taken to assess the LC density and LC morphology (LCM) in a masked fashion. Images were taken 
at the level of sub-basal nerve plexus $(40-60 \mu \mathrm{m}$ from the ocular surface) to describe LC densities. LC densities were examined both at the centre and the corneal periphery at 6 o'clock, according to the examination scheme of Zhivov et al. ${ }^{15}$

Corneal image analysis. Five characteristic images were selected, and the inbuilt semi-automatic system of the device (Heidelberg Eye Explorer software, version 1.5.10.0) was used for calculating cell densities (cell number $/ \mathrm{mm}^{2}$ ). The mean cell number was used in the statistics.

LCM was evaluated on a $0-3$ scale, where a score of 1 represented LCs without dendrites, a score of 2 was given to LCs with small processes, and a score of 3 represented LCs with long processes. Zero (0) depicted the condition when the area of interest was devoid of LCs. Each LC was scored and the mean was calculated to describe the maturation of the LCs at both locations of the cornea (Figure 1).

\section{Statistical analysis}

Descriptive estimates are presented as medians with their respective interquartile ranges. Comparisons between healthy individuals and AS patients were made with Mann-Whitney tests. In case of comparisons between healthy controls and AS patient subgroups, the Kruskal-Wallis test was performed $(P$-values obtained from both). Correlation analyses between clinical variables were carried out with Spearman's index of linear correlation. For all tests, estimates with a $P$-value less than 0.05 were deemed statistically significant.

\section{Results}

\section{Dry eye-related parameters}

The results of functional assays can be seen in Table 2 . Of note, tear production was significantly reduced, and OSDI score was greater in AS patients than in healthy

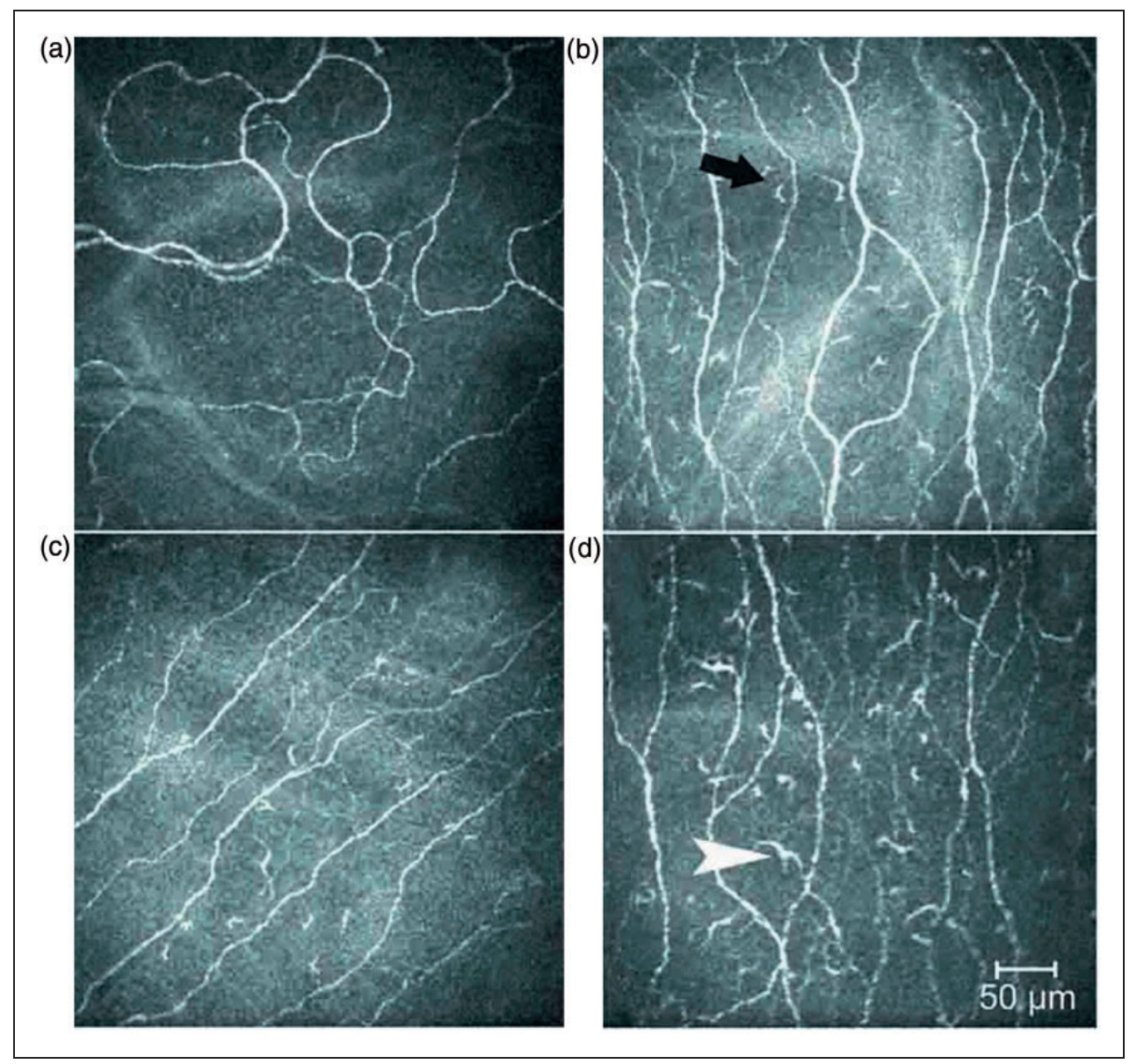

Figure I. Confocal microscopic image of the corneal centre $(a, b)$ and the periphery $(c, d)$ in the control and patient groups. Note that the corneal centre is devoid of LCs in the control group (a), while in the periphery some non-activated and a few activated LCs (LCM I, 2, 3) can be seen (c). (b) Corneal centre in the patient group, where LCs are seen mainly with small processes (black arrow indicates a score of 2 LC), while d represents LCs in the patient group with long processes (white arrowhead pointing at a score of 3 LC). The LCs in the periphery indicate the mature form of LCs in the AS group. 
individuals $(P<0.05)$. OSDI score was also significantly greater in patients positive for human leukocyte antigen $H L A-B 27$ than in controls.

Further analyses in AS subgroups showed decreased tear production in patients with a C-reactive protein (CRP) level of $>5 \mathrm{mmol} / \mathrm{l}$ compared with patients with a CRP level of $<5 \mathrm{mmol} / \mathrm{l}$. However, there was no difference in patients with a CRP level of $<5 \mathrm{mmol} / 1$ compared with controls and in patients with a BASDAI level of $>4(P>0.05)$. There was no further difference between AS subgroups formed according to the therapy.

\section{Confocal microscopy}

Of note, central and peripheral corneal LC density, as well as central LCM in the AS group, were significantly greater than in control group $(P<0.05)$ (Table 3$)$. Further analyses in AS subgroups showed increased LC density in both groups with low and high disease

Table 2. Dry eye-related parameters in different subgroups of AS patients.

\begin{tabular}{|c|c|c|c|c|}
\hline Study group & OSDI & LIPCOF & TBUT $[\mathrm{s}]$ & Schirmer (mm/5 min) \\
\hline Healthy individuals & $6.80[4.20-12.23]$ & $1.00[1.00-1.00]$ & $13.00[\mid 1.0-14.00]$ & $14.00[12.00-15.00]$ \\
\hline All AS patients & $20.80 *[10.40-38.59]$ & $1.00[1.00-1.00]$ & $12.00[7.50-15.00]$ & $5.50 *[1.00-15.75]$ \\
\hline \multicolumn{5}{|c|}{ AS patients according to BASDAI score } \\
\hline$\leq 4(7 \mid \%)$ & $20.80 *[10.40-33.7 I]$ & $1.00[0.00-1.00]$ & $12.00[11.0-15.00]$ & $12.00[1.00-17.50]$ \\
\hline$>4(29 \%)$ & $31.30 *[6.25-45.50]$ & $1.00[1.00-2.00]$ & $9.00[6.00-15.00]$ & $2.00 *[1.00-7.00]$ \\
\hline \multicolumn{5}{|c|}{ AS patients according to CRP } \\
\hline$\leq 5(33 \%)$ & $19.78[10.93-24.43]$ & $0.50[0.00-1.00]$ & $14.00[1|.25-2| .0]$ & $16.00[13.50-22.00]$ \\
\hline$>5(67 \%)$ & $27.07 *[10.40-45.06]$ & $1.00[1.00-1.00]$ & $11.00[6.25-14.50]$ & $1.00 * * *[1.00-5.75]$ \\
\hline \multicolumn{5}{|c|}{ AS patients according to HLA-B27 positivity } \\
\hline Positive $(50 \%)$ & $27.00 *[14.06-42.65]$ & $1.00[1.00-1.00]$ & $12.00[8.00-16.00]$ & $9.00[1.00-16.50]$ \\
\hline Negative (50\%) & $15.60[3.12-36.32]$ & $1.00[0.25-1.75]$ & $12.00[7.50-15.00]$ & $4.00[1.00-15.00]$ \\
\hline \multicolumn{5}{|c|}{ AS patients with history of uveitis } \\
\hline Yes $(29 \%)$ & $31.30 *[10.40-54.12]$ & $1.00[1.00-1.00]$ & $12.00[9.00-22.00]$ & $13.00[1.00-18.00]$ \\
\hline No $(7 \mid \%)$ & $20.80 *[10.40-37.09]$ & $1.00[0.50-1.50]$ & $12.00[7.00-15.00]$ & $2.00[1.00-15.00]$ \\
\hline
\end{tabular}

Data are expressed as median [interquartile range]. Comparisons between healthy individuals and AS patients were made with Mann-Whitney tests. In case of comparisons between healthy controls and AS patient subgroups, a Kruskal-Wallis test was performed. $* P<0.05$ vs healthy individuals; $* * P<0.05$ vs $C R P \leq 5$.

Table 3. Confocal microscopy results in different subgroups of AS patients.

\begin{tabular}{|c|c|c|c|c|}
\hline Study group & LC centr. (cell/mm²) & LC periph. (cell/ $/ \mathrm{mm}^{2}$ ) & LCM centr. & LCM periph. \\
\hline Healthy individuals & $14.50[0.00-35.10]$ & $65.50[46.75-88.00]$ & $1.00[0.70-1.00]$ & $2.00[2.00-3.00]$ \\
\hline All AS patients & $75.50 *[51.18-112.6]$ & $131.0 *[80.33-168.4]$ & $2.00 *[1.00-2.00]$ & $3.00[2.00-3.00]$ \\
\hline \multicolumn{5}{|c|}{ AS patients according to BASDAI score } \\
\hline$\leq 4(7 \mid \%)$ & $69.30 *[45.70-104.0]$ & $108.0 *[78.75-137.4]$ & $2.00 *[1.00-2.00]$ & $2.00[2.00-3.00]$ \\
\hline$>4(29 \%)$ & $92.50 *[56.50-113.0]$ & $170.0 *[135.5-204.0]$ & $1.00[1.00-2.00]$ & $3.00[3.00-3.00]$ \\
\hline \multicolumn{5}{|c|}{ AS patients according to CRP } \\
\hline$\leq 5(33 \%)$ & $66.15^{*}[43.85-107.5]$ & $136.2 *[93.60-197.8]$ & $1.50[1.00-2.00]$ & $2.50[2.00-3.00]$ \\
\hline$>5(67 \%)$ & $79.25^{*}[56.95-115.6]$ & $121.4^{*}[79.63-157.2]$ & $2.00 *[1.00-2.00]$ & $3.00[2.00-3.00]$ \\
\hline \multicolumn{5}{|c|}{ AS patients according to $H L A-B 27$ positivity } \\
\hline Positive $(50 \%)$ & $75.50 *[59.48-107.9]$ & $140.5 *[88.95-197.8]$ & $2.00 *[1.00-2.00]$ & $3.00[2.00-3.00]$ \\
\hline Negative (50\%) & $74.65^{*}[43.85-117.7]$ & $98.40 *[79.63-136.6]$ & $1.50 *[1.00-2.00]$ & $2.50[2.00-3.00]$ \\
\hline \multicolumn{5}{|c|}{ AS patients with history of uveitis } \\
\hline Yes $(29 \%)$ & $111.2 *$ [72.00-129.7] & 143.3* [109.8-204.0] & $2.00 *[2.00-2.00]$ & $3.00[2.00-3.00]$ \\
\hline No $(7 \mid \%)$ & $60.30 *[34.65-91.75]$ & $108.0 *[79.75-153.9]$ & $1.00 *[1.00-2.00]$ & $3.00[2.00-3.00]$ \\
\hline
\end{tabular}

Data are expressed as median [interquartile range]. Comparisons between healthy individuals and AS patients were made with Mann-Whitney tests. In case of comparisons between healthy controls and AS patient subgroups, a Kruskal-Wallis test was performed. $* P<0.05$ vs healthy individuals. 
activity, as assessed by the BASDAI index and the serum CRP levels. Correlation analysis showed a strong positive correlation between BASDAI and peripheral LC density $(\mathrm{r}=0.51, P<0.05)$. The activation status of the LCs, as assessed by the central corneal LCM score, increased in patients with CRP levels $>5 \mathrm{mmol} / 1 \mathrm{compared}$ with controls and patients with CRP levels $<5 \mathrm{mmol} / \mathrm{l}$. We found no further difference between AS subgroups formed according to the therapy.

\section{Discussion}

Confocal microscopy, which is capable of visualizing the ultrastructure of the organ examined at a cellular level, has been developed in recent years. Through obtaining coronal optical sections of the transparent part of the eye, we can explore the resident innate immune system of the ocular surface. Under nonpathological circumstances, the majority of LCs reside in the periphery of the cornea, with numbers of LCs tapering rapidly towards the corneal centre. ${ }^{15}$ Upon different stimuli, LCs are capable of transforming into an active participant of corneal immune responses characterized by the formation of dendrite-like processes. ${ }^{16}$ LCs have been shown to play important roles in promoting inflammatory reactions and immunogenity, and have a pivotal role in the regulation of ocular surface immune processes. ${ }^{17}$

To the best of our knowledge, this is the first study to investigate the characteristics of corneal LC density and morphology in AS. We confined our investigation on the density and morphology of corneal LCs to their presumed role in ocular surface diseases and their extraordinary capacity to stimulate naive T-cells. We have demonstrated increased density and activation of dendritic cells in the cornea of AS patients in the absence of clinically manifest ocular inflammation. These novel results support the systemic multi-organ-related feature of AS affecting the eye and are also in line with the results of Wakamatsu ${ }^{18,19}$ and Villani, ${ }^{20}$ who found greater inflammatory cell infiltration of the conjunctiva in SS and in atopic keratoconjunctivitis. It has been demonstrated that the inflammatory cells, and structural changes of the cornea and activation of keratocytes could highlight corneal involvement in local or general conditions. ${ }^{21}$ Similarly to our recent study on RA patients, ${ }^{9}$ we observed an increase in the number and activation of central LCs, which did not appear to be associated with articular inflammatory disease activity; however, the central corneal LCM score was increased in patients with a higher systemic inflammation according to the CRP level, and the peripheral LC density showed positive correlation with BASDAI, which further supports our theory on the corneal influence of AS.

We have further demonstrated that a history of uveitis resulted in abundance of LCs in the cornea. These
LCs expressed no protrusions, suggesting their immunological inactivity. Whether these inactive LCs enhance the tolerogenic wing of innate immune system by producing anti-inflammatory factors to regain the integrity and function of ocular surface is still unknown. The connection between the LC presence and ocular surface disturbances is still controversial and requires further studies in this field.

$H L A-B 27$ is the primary disease susceptibility gene for AS, which occurs in $6-10 \%$ of Caucasians, but is present in more than $90 \%$ of patients with AS and $50 \%$ of patients with acute anterior uveitis. ${ }^{22}$ Their function is not fully understood, but they are the cellular markers of individuality and they play an important role in immunological reactivity at the cellular level. The fact that AS is a heterogeneous disease has been recognized for the past 30 years from the differences between $H L A$ $B 27$-positive and $H L A-B 27$-negative patients. ${ }^{23}$ The aim of this study was not only to investigate the corneal manifestation in AS, but also to clarify the possible role of $H L A-B 27$ status in corneal immunologic reactivity. The prevalence of $H L A-B 27$ in the overall AS patient group in our clinic is more than $90 \%$, which is in accordance with data in literature. ${ }^{22}$ In a second step, we selected a 1:1 ratio pf $H L A$-B27-positive to $H L A-B 27$-negative patients. Laval et al. ${ }^{24}$ showed greater frequency of relapse, severity and complications of uveitis in $H L A-B 27$-positive patients compared with those of $H L A-B 27$-negative patients. Surprisingly, there was no significant difference in selected $H L A$-B27-positive and $H L A-B 27$-negative patients neither in clinical characteristics nor in dry eye-related parameters and confocal microscopic data. However, the number of investigated AS patients is limited; our data suggest that the $H L A-B 27$ status has no influence on corneal immune mechanism.

Therapy-related alterations in tear production and their presumed role on LCs were demonstrated in recent work by our team. ${ }^{9}$ We noted decreased tear production in patients with elevated CRP, representing an active systemic inflammatory status in AS. This observation was confirmed in our preliminary analysis, where the relationship between these parameters had a statistically significant strong positive correlation. Our data are in accordance with the results of Villani et al., ${ }^{25}$ which suggest that these alterations of the cornea are rather associated with the systemic inflammatory effect of the disease than a specific pathogenic differences in dry eye disease. Theoretically, with higher systemic inflammation in AS, one may assume an increase in the secretion of pro-inflammatory cytokines in the tears. Presumably, the higher amount of these cytokines in the tears of AS patients might impair tear production, and it may partly explain the dry eye mechanism without the presence of true overlapping SS. In fact, IL-1, IL- 6 and TNF- $\alpha$ have been found to be overexpressed and to be accumulated in the ocular 
surface in dry eye, ${ }^{26-28}$ and they have a key role in the pathomechanism of $\mathrm{AS}^{29}$ In turn, these cytokines are thought to have the capacity to decrease tear production via neuronal and hormonal effects, ${ }^{28}$ and have been shown to facilitate centripetal migration of LCs. ${ }^{30}$ Our theory can be partly supported by Villani et al., ${ }^{31}$ who showed a higher IL- $1 \alpha$ and IL-6 level in active RA patients with secondary SS, and found lower levels of those cytokines in patients with a reduction of the systemic inflammation; however, the number of investigated patients was limited and they could not prove this pattern in their non-Sjögren RA patient group. ${ }^{31}$ The limitation of our study is that only antiTNF- $\alpha$-treated AS patients were involved. Investigation of anti-TNF- $\alpha$-naive AS patients to see whether the systemic anti-inflammatory drug has an influence on the corneal immune mechanisms would also be interesting. Despite the anti-TNF- $\alpha$ therapy, seven of the 24 AS patients $(29 \%)$ were refractory to the first anti-TNF- $\alpha$ agent and three out of these seven AS patients were already treated with the third antiTNF- $\alpha$ agent. Therefore, this anti-TNF- $\alpha$ non-responder AS group represented a group of patients with particularly high systemic inflammation.

As previous studies demonstrated the heterogeneity of resident corneal immune cells, ${ }^{32}$ we can rather consider the cornea as an active participant in the complexity of interactions between environment and the ocular surface then a non-compliant anatomical surface. ${ }^{9}$ Indeed, the immune privilege is secured through a series of active mechanisms rather than a simple consequence of the avascular and alymphatic nature of the cornea. Additionally, our results support the role of the systemic inflammation in dry eye patomechanism.

Further investigations are required to clarify the role of adaptive immunity in the cornea in systemic inflammatory rheumatic diseases, and the connection between the dry eye mechanism, the systemic inflammation and the LCs. Maintenance of corneal homeostasis relies on complex interactions of various immunomechanisms, where ocular surface LCs might play a key role.

\section{Funding}

Attila Balog and Gergely Toldi were supported by the Magyary Zoltán Hungarian Scientific Scholarship (Grant no. TÁMOP-4.2.4. A/2-11-1-2012-001).

\section{Conflict of interest}

The authors do not have any potential conflicts of interest to declare.

\section{Acknowledgement}

We would like to express our gratitude to $\mathrm{Mr}$ Anwar Musah for his valuable contribution in proofreading the manuscript.

\section{References}

1. Braun J and Sieper J. Ankylosing spondylitis. Lancet 2007; 9570: 1379-1390.

2. Pato E, Muñoz-Fernández S, Francisco F, et al. Uveitis Working Group from Spanish Society of Rheumatology. Semin Arthritis Rheum 2011; 40: 314-323.

3. Linder R, Hoffmann A and Brunner R. Prevalence of the spondyloarthritides in patients with uveitis. $J$ Rheumatol 2004; 31: 2226-2229.

4. Monnet D, Breban M, Hudry C, et al. Ophthalmic findings and frequency of extraocular manifestations in patients with $H L A$ B27 uveitis: a study of 175 cases. Ophthalmology 2004; 111: 802-809.

5. Kobak S, Kobak AC, Kabasakal Y, et al. Sjögren's syndrome in patients with ankylosing spondylitis. Clin Rheumatol 2007; 26: 173-175.

6. Van der Horst-Bruinsma IE and Nurmohamed MT. Management and evaluation of extra-articular manifestations in spondyloarthritis. Ther Adv Musculoskelet Dis 2012; 4: 413-422.

7. Schaumburg CS, Siemasko KF, De Paiva CS, et al. Ocular surface APCs are necessary for autoreactive T cell-mediated experimental autoimmune lacrimal keratoconjunctivitis. J Immunol 2011; 187: 3653-3662.

8. Lin H, Li W, Dong N, et al. Changes in corneal epithelial layer inflammatory cells in aqueous tear-deficient dry eye. Invest Ohpthalmol Vis Sci 2010; 51: 122-128.

9. Marsovszky L, Resch MD, Németh J, et al. In vivo confocal microscopic evaluation of corneal Langerhans cell density, and distribution and evaluation of dry eye in rheumatoid arthritis. Innate Immun. Epub ahead of print. 30 November 2012.

10. Van der Linden S, Valkenburg HA and Cats A. Evaluation of diagnostic criteria for ankylosing spondylitis. A proposal for modification of the New York criteria. Arthritis Rheum 1984; 27: $361-368$.

11. Garrett S, Jenkinson T, Kennedy LG, et al. A new approach to defining disease status in ankylosing spondylitis: the Bath Ankylosing Spondylitis Disease Activity Index. J Rheumatol 1994; 21: 2286-2291.

12. Vitali C, Bombardieri S, Jonsson R, et al. Classification criteria for Sjögren's syndrome: a revised version of the European criteria proposed by the American-European Consensus Group. Ann Rheum Dis 2002; 61: 554-558.

13. Németh J, Fodor E, Lang Z, et al. Lid-parallel conjunctival folds (LIPCOF) and dry eye: a multicentre study. $\mathrm{Br} J$ Ophthalmol 2012; 96: 1380-1385.

14. Pult H, Purslow C and Murphy PJ. The relationship between clinical signs and dry eye symptoms. Eye 2011; 25: 502-510.

15. Zhivov A, Stave J, Vollmar B and Guthoff R. In vivo confocal microscopic evaluation of Langerhans cells density and distribution in the normal corneal epithelium. Graefe's Arch Clin Exp Ophthalmol 2005; 243: 1056-1061.

16. Hamrah P, Huq OS, Liu Y, et al. Corneal immunity is mediated by heterogenous population of antigen-presenting cells. J Leukoc Biol 2003; 73: 172-178.

17. Chen W, Lin H, Dong N, et al. Cauterization of central cornea induces recruitment of major histocompatibility complex class II + Langerhans cells from limbal basal epithelium. Cornea 2010; 29: 73-79.

18. Wakamatsu TH, Sato EA, Matsumoto Y, et al. Conjunctival in vivo confocal scanning laser microscopy in patients with Sjögren syndrome. Invest Ophthalmol Vis Sci 2010; 51: 144-150.

19. Wakamatsu TH, Okada N, Kojima T, et al. Evaluation of conjunctival inflammatory status by confocal scanning laser microscopy and conjunctival brush cytology in patients with atopic keratoconjunctivitis (AKC). Mol Vis 2009; 15: 1611-1619. 
20. Villani E, Beretta S, Galimberti D, et al. In vivo confocal microscopy of conjunctival roundish bright objects: young, older, and Sjögren subjects. Invest Ophthalmol Vis Sci 2011; 52: 4829-4832.

21. Villani E, Viola F, Sala R, et al. Corneal involvement in Graves' orbitopathy: an in vivo confocal study. Invest Ophthalmol Vis Sci 2010; 51: 4574-4578.

22. Khan MA, Kushner I and Braun WE. Comparison of clinical features of $H L A-B 27$ positive and negative patients with ankylosing spondylitis. Arthritis Rheum 1977; 20: 909-912.

23. Khan MA. HLA-B27 and its pathogenic role. J Clin Rheumatol 2008; 14: 50-52.

24. Laval SH, Timms A, Edwards S, et al. Whole-genome screening in ankylosing spondylitis: evidence of non-MHC genetic-susceptibility loci. Am J Hum Genet 2001; 68: 918-926.

25. Villani E, Galimberti D, Viola F, et al. Corneal involvement in rheumatoid arthritis: an in vivo confocal study. Invest Ophthalmol Vis Sci 2008; 49: 560-564.

26. Boehm N, Riechardt AI, Wiegand M, et al. Proinflammatory cytokine profiling of tears from dry eye patients by means of Ab microarrays. Invest Ophthalmol Vis Sci 2011; 52: 7725-7730.
27. Lam H, Bleiden L, de Paiva CS, et al. Tear cytokine profiles in dysfunctional tear syndrome. Am J Ophthalmol 2009; 147: 198-205.

28. Massingale ML, Li X, Vallabhajosyula M, et al. Analysis of inflammatory cytokines in the tears of dry eye patients. Cornea 2009; 28: 1023-1027.

29. Goh L and Samanta A. Update on biologic therapies in ankylosing spondylitis: a literature review. Int J Rheum Dis 2012; 15 : 445-454.

30. Dana Reza, Dai Ruoping, Zu Shuning, et al. Interleukin-1 receptor antagonist suppresses Langerhans cell activity and promotes ocular immune privilege. Invest Ophthalmol Vis Sci 1998; 39: 70-77.

31. Villani E, Galimberti D, Del Papa N, et al. Inflammation in dry eye associated with rheumatoid arthritis: Cytokine and in vivo confocal microscopy study. Innate Immun. Epub ahead of print 22 January 2013.

32. Hamrah $\mathrm{P}$ and Dana MR. Corneal antigen-presenting cells. Chem Immunol Allergy 2007; 92: 58-70. 